\title{
Prótese total do cotovelo: análise retrospectiva a médio prazo dos 10 primeiros casos
}

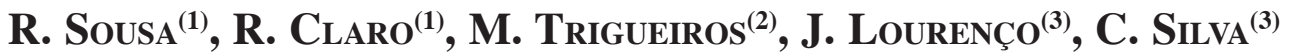 \\ Serviço de Ortopedia. Hospital de Santo António - Centro Hospitalar do Porto. Portugal \\ (I) INTERNO COMPLEMENTAR \\ (2) ASSISTENTE HOSPITALAR \\ (3) Assistente GRaduado
}

Correspondência:

Dr. Ricardo Sousa

Serviço de Ortopedia. Hospital de Santo António - Centro Hospitalar do Porto

Largo Professor Abel Salazar

4099-001. Porto. Portugal

Telefono 222077500

Fax 222087632

e-mail: ricardojgsousa@gmail.com

Actualmente, a artrite reumatóide e a artrose pós traumática constituem as principais indicações para a realização de uma artroplastia total do cotovelo. O objectivo deste estudo é analisar os resultados a médio prazo da experiência inicial na nossa instituição. Secundariamente quisemos procurar diferenças entre próteses não-constritivas e as semi-constritivas. Para tal foram revistos retrospectivamente dez casos consecutivos (oito de artrite reumatóide e dois de artrose pós-traumática) correspondentes a sete mulheres e dois homens com idade média de 48 anos com um seguimento médio de 34 meses. O valor médio da escala visual da dor baixou significativamente, de 7.9 para $2.4(\mathrm{t}=3.145 ; \mathrm{p}=0.012)$, após a cirurgia. A flexão média foi $118.5^{\circ}(\mathrm{r}=85-145)$ com défice de extensão médio de $24^{\circ}(\mathrm{r}=5-50)$; pronação média de $84^{\circ}(\mathrm{r}=75-90)$ e supinação $80.5^{\circ}(\mathrm{r}=30-90)$. Em termos funcionais o Mayo Elbow Performance Score médio foi $87.5(\mathrm{r}=55-100)$. Entre os dois conceitos de próteses utilizados a única diferença significativa encontrada foi um menor arco de mobilidade nas próteses não-constritivas: $77.5^{\circ}$ vs $105.8^{\circ}$ $(\mathrm{t}=5.143 ; \mathrm{p}=0.014)$. Este estudo confirma a artroplastia total do cotovelo como uma opção válida na nossa instituição.
Rheumatoid and post-traumatic arthritis are the most common present indications for total elbow arthroplasty. The goal of this study is to analyze the midterm results of our institution's initial experience. Secondarily, we wanted to search for differences between unconstricted and semi-constricted prosthesis. In that regard we retrospectively reviewed ten consecutive cases (eight rheumatoid and two posttraumatic arthritis) corresponding to seven women and two men with an average age of 48 years-old and a average follow-up of 34 months. Visual Ana$\log$ Pain Scale improved significantly after surgery -7.9 to $2.4(\mathrm{t}=3.145 ; \mathrm{p}=0.012)$. Average elbow flexion found was $118.5^{\circ}(\mathrm{r}=85-145)$ with an extension deficit of $24\left(5^{\circ}-50^{\circ}\right)$; average pronation was $84^{\circ}$ $\left(\mathrm{r}=75^{\circ}-90^{\circ}\right)$ and supination $80.5^{\circ}(\mathrm{r}=30-90)$. The average Mayo Elbow Performance Score was 87.5 $(r=55-100)$. The only significant difference found between the two different prosthesis concepts was a lower mobility in the unconstricted group: $77.5^{\circ}$ vs $105.8^{\circ}(\mathrm{t}=5.143 ; \mathrm{p}=0.014)$. This study confirms that total elbow arthroplasty as a valid option in our institution. 


\section{INTRODUÇÃO}

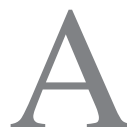

s principais indicações para artroplastia total do cotovelo são a artrite reumatóide e a artrose pós traumática ${ }^{1,2}$. Outras patologias como osteoartrose primária, fracturas, artropatia hemofílica, sequelas de artrite séptica ou mesmo ressecção tumoral podem constituir indicações para substituição protésica do cotovelo ${ }^{3}$. Num doente jovem com elevada exigência funcional ou pouco motivado, a artroplastia deve ser preterida em relação a outras opções ${ }^{1,2}$ No entanto, em doentes idosos, a artroplastia total pode mesmo ser uma alternativa melhor que a osteossíntese em fracturas intra-articulares distais do úmero ${ }^{4}$.

A modificação do nível de actividade deve sempre ser considerada antes se optar pelo tratamento cirúrgico ${ }^{3}$. Dependendo da etiologia e ainda da idade e exigência funcional do doente, as opções cirúrgicas podem incluir a sinovectomia aberta ou artroscópica ${ }^{5,6}$ ou o desbridamento articular com limpeza dos osteófitos (operação de Outerbridge) aberto ou artroscópico ${ }^{7,8}$. Outras opções como artroplastia de interposição ${ }^{9}$ ou hemiartroplastia radiocapitelar ${ }^{10}$ têm vindo a ser estudadas recentemente. Não obstante todas estas opções, a artroplastia total do cotovelo é a opção mais definitiva para o alívio da dor, melhoria do arco de mobilidade ou tratamento da instabilidade dolorosa em casos de artrose avançada ${ }^{11}$.

O objectivo deste estudo foi o de analisar os resultados obtidos a médio prazo em termos de alívio da dor, arco de mobilidade e resultados funcionais conseguidos, taxa de complicações e grau de satisfação dos doentes numa série inicial de 10 artroplastias totais do cotovelo consecuti- vas, realizadas na nossa instituição. Outro objectivo foi comparar os dois conceitos de próteses utilizados: próteses não-constritivas $\left(\mathrm{IBP}^{\circledR}-\right.$ Biomet) e semi-constritivas (GSB III ${ }^{\circledR}-$ Zimmer e Latitude $^{\circledR}-$ Tornier).

\section{MATERIAL E MÉTODO}

Foram avaliadas, retrospectivamente, dez artroplastias totais do cotovelo efectuadas em nove doentes entre 2003 e 2007. As indicações para cirurgia foram: artrite reumatóide em sete doentes (oito cotovelos) e artrose pós-traumática em dois casos. A população estudada incluiu 7 mulheres e 2 homens com idade média de 48 anos $(r=33-77)$. Foram operados 6 cotovelos esquerdos e 4 direitos, sendo que apenas em 4 casos se tratava do membro dominante. O seguimento médio foi 34 meses com um mínimo de 12 e um máximo de 65 meses.

As próteses utilizadas na nossa série foram a $\operatorname{IBP}^{\circledR}(n=4)$, a GSB III ${ }^{\circledR}(n=5)$ e a Latitude ${ }^{\circledR}$ $(\mathrm{n}=1)$. A Latitude foi implantada utilizando a opção semi-constritiva (Figura 1). Embora as cirurgias tenham sido realizadas por três cirurgiões diferentes, foi utilizada a mesma técnica cirúrgica: «triceps splitting» com elevação de flaps osteoperiostais segundo a técnica de Gshwend ${ }^{12}$, transposição anterior do nervo cubital e cimentação de ambos os componentes (com excepção da haste umeral da IBP em doentes jovens). Nas primeiras duas semanas após a cirurgia os doentes são autorizados a iniciar mobilização passiva iniciando mobilização activa e reabilitação fisiátrica na terceira semana.

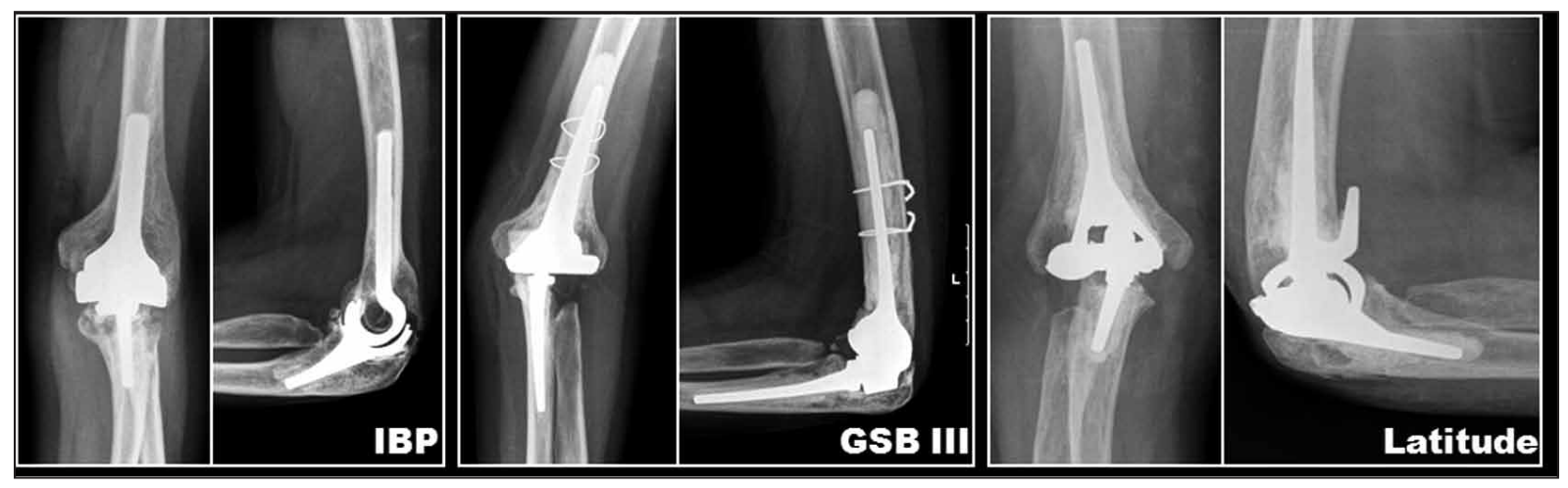

Figura 1. Aspecto radiológico das diferentes próteses utilizadas 
Os doentes foram avaliados clinicamente utilizando diferentes escalas de avaliação subjectiva e objectiva: Mayo Elbow Performance Score (MEPS) $)^{13}$; Hospital for Special Surgery elbow scoring system (HSS $)^{14}$ e Disabilities of the Arm, Shoulder and Hand (DASH) ${ }^{15}$. Foram especificamente avaliados a progressão da dor entre o pré e o pós-operatório e o arco de mobilidade conseguido. Para além da avaliação clínica, foram registadas todas as complicações ocorridas. A avaliação da descelagem foi feita de acordo com o sistema descrito por Little et al. ${ }^{11}$, radiolucência com sintomatologia adequada, radiolucência $\geq 2 \mathrm{~mm}$, ou migração de componente.

Foi aplicado o teste t para apreciar diferenças de valores médios entre os diversos parâmetros e sub-grupos utilizando para isso o PASW Statistics 17 (SPSS Inc.).

\section{RESULTADOS}

O alívio da dor foi significativo em quase todos os doentes, passando de uma média de 7.9 para $2.4(\mathrm{t}=3.145 ; \mathrm{p}=0.012)$ na escala visual analógica (VAS) da dor entre o pré e o pós-operatório. Apenas se registou um caso de agravamento das queixas álgicas que corresponde ao caso em que se verificou infecção da prótese.
Os valores de mobilidade registados na consulta de revisão foram: flexão média de $118.5^{\circ}$ $(\mathrm{r}=85-145)$ com um défice de extensão médio de $24^{\circ}(\mathrm{r}=5-50)$; pronação média de $84^{\circ}\left(\mathrm{r}=75^{\circ}\right.$ $\left.90^{\circ}\right)$ e supinação $80.5^{\circ}(\mathrm{r}=30-90)$ (Figura 2). As avaliações objectivas e funcionais registadas durante a consulta de revisão foram: MEPS médio $87.5(\mathrm{r}=55-100)$, HSS médio $80.6(\mathrm{r}=67-91)$ e DASH médio $51.2(\mathrm{r}=20.5-78.4)$. A tabela 1 mostra, de forma resumida, os resultados obtidos de acordo com o diagnóstico pré-operatório e tipo de implante utilizado.

Utilizando os critérios definidos a priori para análise radiológica apenas se detectou um caso de descelagem (séptica) com migração de ambos os componentes. Foram no entanto detectados mais dois casos com osteólise no componente cubital, ambos com próteses não constritivas (IBP). Num deles, a zona radiolucente rodeando o componente cubital é superior a $2 \mathrm{~mm}$ pelo que se pode considerar um caso de descelagem. Em termos de estabilidade não se registou nenhum caso de luxação e nenhum cotovelo foi considerado muito instável. No entanto, 6 dos 10 cotovelos foram classificados como moderadamente instáveis com base na avaliação clínica durante a consulta de revisão. Em dois casos houve fractura intra-operatória da extremidade distal do úmero (ambos com prótese GSB III).

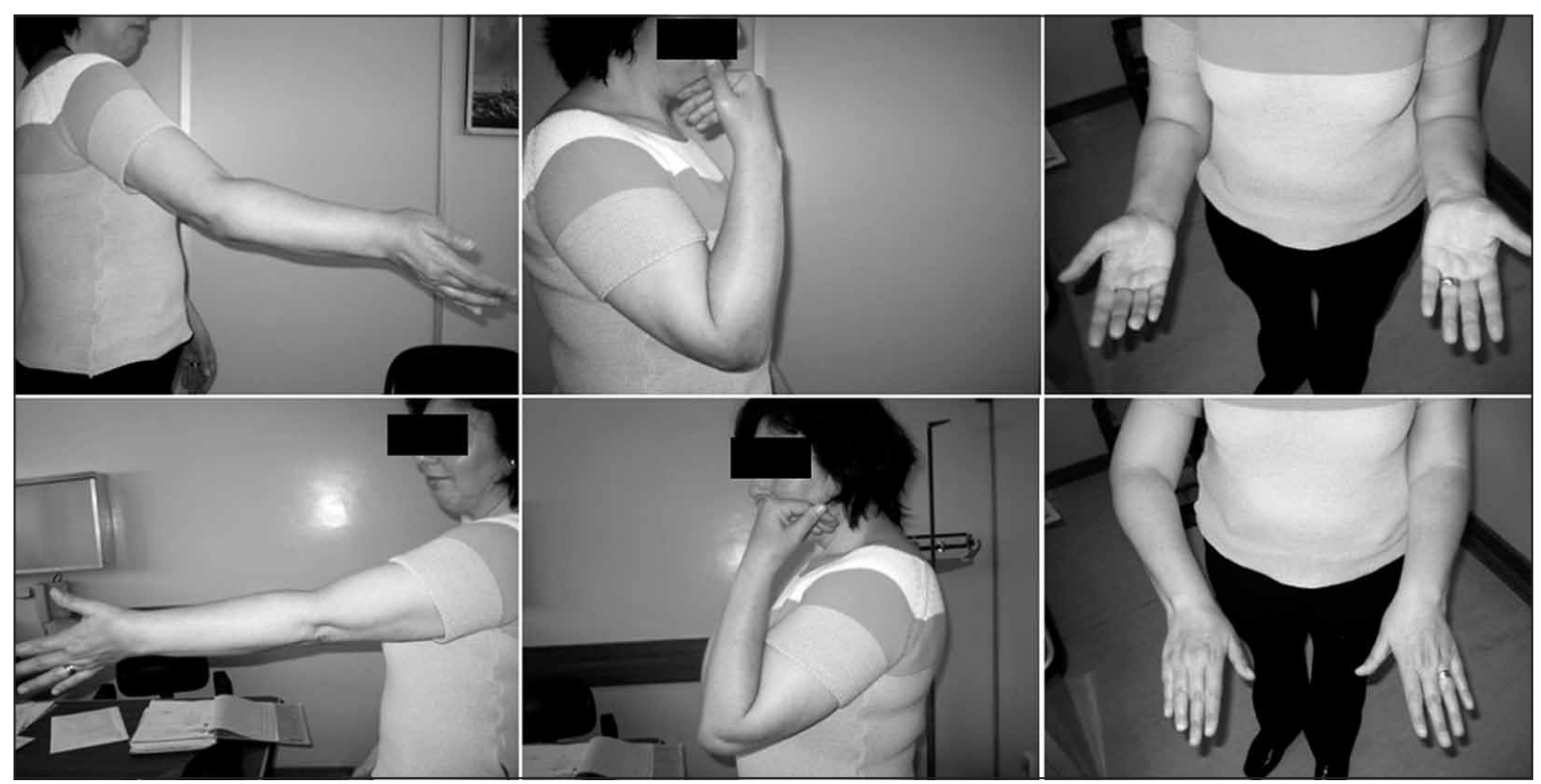

Figura 2. Mobilidade articular em doente com prótese do cotovelo bilateral. 
Tabela I - RESULTADOS DE ACORDO COM O DIAGNÓSTICO PRÉ-OPERATÓRIO E TIPO DE IMPLANTE

\begin{tabular}{|c|c|c|c|c|c|}
\hline & Diagnóstico * & Tipo de Implante & $\Delta$ Dor & Arco mobilidade & MEPS \\
\hline$\# 1$ & AR estadio IV & GSB III & -9 & $105^{\circ}$ & 95 \\
\hline$\# 2$ & AR estadio IIIA & IBP & -8 & $35^{\circ}$ & 80 \\
\hline$\# 3$ & AR estadio II & IBP & -7 & $80^{\circ}$ & 90 \\
\hline$\# 5$ & AR estadio IIIA & IBP & 0 & $105^{\circ}$ & 100 \\
\hline$\# 6$ & AR estadio IIIA & GSB III & +2 & $90^{\circ}$ & 90 \\
\hline$\# 7$ & AR estadio IIIB & GSBIII & -9 & $120^{\circ}$ & 55 \\
\hline$\# 8$ & Pós-traumática & GSB III & -4 & $100^{\circ}$ & 95 \\
\hline$\# 9$ & AR estadio IIIA & GSB III & -8 & $115^{\circ}$ & 90 \\
\hline$\# 10$ & AR estadio II & Latitude & -6 & $125^{\circ}$ & 80 \\
\hline
\end{tabular}

* Estadio de AR segundo classificação de cotovelo reumatóide da Clinica Mayo ${ }^{23}$

$\Delta$ Dor - variação da escala visual analógica da dor (VAS) entre o pré e o pós-operatório: (-) melhoria; (+) agravamento MEPS - Mayo Elbow Performance Score

Contudo, ambas foram resolvidas com a adição de cerclage com fios de aço sem consequências importantes. Apenas se registou um caso de neuropatia do cubital que resolveu completamente ao fim de 12 meses e nenhum caso de rotura do tríceps. Uma das próteses infectou o que justifica a única re-intervenção (extracção da prótese). Não se registou qualquer falência do material.

Sete dos nove pacientes responderam estar satisfeitos/muito satisfeitos com o procedimento e apenas dois deles (infecção e a neuropatia do cubital) não o recomendaria a alguém na mesma situação.

Comparando os resultados entre os dois conceitos de próteses utilizado, é interessante verificar que o arco de mobilidade médio é significativamente inferior $(\mathrm{t}=5.143 ; \mathrm{p}=0.014)$ no grupo das próteses não constritivas (IBP) quando comparado com o grupo das próteses semi-constritivas (GSB III + Latitude): $77.5^{\circ}$ vs $105.8^{\circ}$ (para uma média global de $94.5^{\circ}$ ). No entanto em termos de resultados funcionais, não se registaram diferenças importantes em qualquer escala de avaliação. Curiosamente não se verifica diferenças relevantes entre o grupo de próteses não constritivas (IBP) e as semi-constritivas (GSB III) no que respeita a avaliação da instabilidade.

\section{DISCUSSÃO}

A indicação para artroplastia total do cotovelo é o alívio da dor e/ou melhoria da mobilidade. O nosso principal objectivo foi avaliar em que medida a realização de uma prótese do cotovelo na nossa instituição satisfaz estas condições.

Este estudo tem várias limitações. A primeira é inerente ao facto de se tratar de um estudo retrospectivo de um único centro. Em segundo lugar, trata-se de um número pequeno de doentes e o facto de terem sido utilizados dois conceitos de próteses diferentes introduz uma dificuldade acrescida na interpretação dos resultados. Nu- 
ma fase inicial o critério de escolha entre próteses semi-constritivas e não-constritivas foi a presença ou não de instabilidade pré-operatória. Com o acumular da experiência, observamos uma tendência favorável às próteses semi-constritivas pelo que actualmente é essa a nossa primeira escolha. Por fim, mas não menos importante, é o facto de os resultados reflectirem a fase inicial da curva de aprendizagem dos cirurgiões envolvidos.

A melhoria da dor que se constatou na nossa série com um seguimento médio de quase três anos, confirma aquela que é uma das principais virtudes deste procedimento. É de esperar um alívio significativo da dor a longo prazo ${ }^{11}$.

O nível funcional obtido (MEPS 87.5) está de acordo com os resultados publicados que variam entre 87-94 para artrite reumatóide e 84-95 para artrose pós-traumática ${ }^{16}$.

Tradicionalmente a artroplastia total do cotovelo tem sido associada a uma alta taxa de complicações ${ }^{12,17}$. Ela deriva da complexidade inerente à articulação do cotovelo, ao seu pobre revestimento de partes moles, à sua íntima relação com o nervo cubital mas também com o elevado perfil de risco da população-alvo (ex: artrite reumatóide). A definição de descelagem é bastante variável na literatura o que afecta a interpretação dos resultados ${ }^{11}$. O componente cubital, especialmente se não cimentado parece estar em maior risco ${ }^{18,19}$. Teoricamente existe uma relação directa entre estabilidade intrínseca do implante e descelagem que se deve à maior ou menor concentração de forças na interface osso-cimento. No entanto, a nossa série revelou maior preponderância de descelagem asséptica do componente cubital das próteses não constritivas. Os autores especulam que este facto poderá estar relacionado com o maior seguimento médio quando comparado com o das próteses semi-constritivas (47 vs 26 meses). A instabilidade inclui não só a luxação grosseira, mas também sinais radiográficos de subluxação ou sintomas subjectivos ${ }^{11}$. De acordo com Gregory et al. ${ }^{2}$ como seria de esperar a taxa é superior nas próteses não-constritivas $(6 \%)$ do que nas semiconstritivas (1\%). Em cotovelos instáveis, como na artrite reumatóide, esta diferença pode ser importante. A taxa de neuropatia do cubital perma- nente, sensitiva ou motora, segundo Tashjian et al. ${ }^{8}$, ronda os $5 \%$. Esta afirmação é, no entanto, algo controversa uma vez que a maioria dos estudos não especifica o estado pré-operatório do nervo cubital. É um facto que a disfunção do nervo cubital não é incomum no pré-operatório de doentes candidatos a artroplastia do cotovelo ${ }^{20}$. A taxa de infecção protésica no cotovelo, segundo Gregory et al. ${ }^{2}$, ronda também os 4-5\%, longe do alarmismo inicial que rodeou este tipo de artroplastia. De salientar que mesmo perante uma infecção, é possível tratá-la com desbridamento agressivo e antibioterapia adequada ou levar a cabo uma revisão em dois tempos alcançando uma prótese funcionante livre de infecção ${ }^{21}$. Na nossa série encontramos uma proporção maior que a descrita de infecção (1/10). Contudo, nenhuma inferência foi feita, uma vez que os autores julgam tratar-se apenas de um efeito estatístico devido ao pequeno tamanho da amostra.

De acordo com o que já foi sugerido na literatura $^{11}$, parece haver maior mobilidade com as próteses semi-constritivas do que as não-constritivas facto que pudemos confirmar na nossa série. No entanto este achado paradoxal não está ainda totalmente explicado nem tão pouco é consensual uma vez que outros autores como Wright et al. ${ }^{22}$ não confirmaram esta tendência. Ainda de acordo com a revisão da literatura feita por Little et al. ${ }^{11}$, os resultados funcionais parecem ser melhor com as próteses semi-constritivas do que com as não-constritivas sendo que as totalmente constritivas são as que têm piores resultados. $\mathrm{Na}$ nossa série não foi possível encontrar diferenças entre os dois conceitos utilizados no que respeita a função.

Os resultados obtidos estão de acordo com a literatura internacional e sugerem que a artroplastia total do cotovelo é uma alternativa eficaz para aliviar de forma significativa a dor e promover uma articulação estável com boa mobilidade com uma taxa aceitável de complicações. As próteses semi-constritivas parecem permitir maior arco de mobilidade com maior estabilidade do cotovelo. Esta é uma série pequena da qual não se podem retirar conclusões definitivas. Pretende apenas reflectir sobre aquela que é foi a experiência inicial na nossa instituição e que é suficiente para nos encorajar a continuar. 


\section{BIBLIOGRAFIA}

1. Gallo RA, Payatakes A, Sotereanos DG. Surgical options for the arthritic elbow. J Hand Surg Am, 2008; 33: 746-59.

2. Gregory JJ, Ennis O, Hay SM. Total elbow arthroplasty. Current Orthop, 2008; 22: 80-9.

3. Kokkalis ZT, Schmidt CC, Sotereanos DG. Elbow arthritis: current concepts. J Hand Surg Am, 2009; 34: 761-68.

4 -McKee MD, Veillette CJH, Hall JA et al. A multicenter, prospective, randomized, controlled trial of open reduction-internal fixation versus total elbow arthroplasty for displaced intra-articular distal humeral fractures in elderly patients. J Shoulder Elbow Surg, 2009; 18: 3-12.

5. Fuerst M, Fink B, Ruther W. Survival analysis and longterm results of elbow synovectomy in rheumatoid arthritis. J Rheumatol, 2006; 33: 892-6.

6. Tanaka N, Sakahashi H, Hirose K, et al. Arthroscopic and open sinovectomy of the elbow in rheumatoid arthritis. J Bone Joint Surg Am, 2006; 88: 521-5.

7. Adams JE, Wolff LH III, Merten SM, et al.. Osteoarthritis of the elbow: results of arthroscopic osteophyte resection and capsulectomy. J Shoulder Elbow Surg, 2008; 17: 126-31.

8. Tashjian RZ, Wolf JM, Ritter M, et al. Functional outcomes and general health status after ulnohumeral arthroplasty for primary osteoarthritis of the elbow. J Shoulder Elbow Surg, 2006; 15: 357-66.

9. Larson AN, Morrey BF. Interposition arthroplasty with an Achilles tendon allograft as a salva- ge procedure for the elbow. Bone Joint Surg Am, 2008; 90 : 2714-23.

10. Heijink A, Morrey BF, Cooney WP III. Radiocapitellar hemiarthroplasty for radiocapitellar arthritis: a report of three cases. J Shoulder Elbow Surg, 2008; 17: e12-e15.

11. Little CP, Graham AJ, Carr AJ. Total elbow arthroplasty. J Bone Joint Surg Br 2005; 87: 43744.

12. Gshwend N, Simmen BR, Matejovsky Z. Late complications in elbow arthroplasty. J Shoulder Elbow Surg, 1996; 5: 8696.

13. Morrey BF, An KN, Chao EYS. Functional evaluation of the elbow. In: Morrey BF, ed. The elbow and its disorders, $2^{\text {nd }}$ edn. Philadelphia, PA, USA: W.B. Saunders, 1993: 86-97.

14. Figgie MP, Inglis AE, Mow CS, et al. Total elbow arthroplasty for complete ankylosis of the elbow. J. Bone and Joint Surg Am, 1989; 71: 513-20.

15. Beaton DE, Katz JN, Fossel $\mathrm{AH}$, et al. Measuring the whole or the parts? Validity, reliability and responsiveness of the disabilities of the arm, shoulder and hand outcome measure in different regions of the upper extremity. J Hand Ther, 2001; 14: 128-46.

16. Shi LL, Zurakowski D, Jones DG, et al. Semiconstrained primary and revision total elbow arthroplasty with use of the Coonrad-Morrey prosthesis. J Bone Joint Surg Am, 2007; 89: 1467-75.

17. Tachihara A, Nakamura H, Yoshioka $\mathrm{T}$, et al. Postoperative results and complications of total elbow arthroplasty in patients with rheumatoid arthritis: three types of nonconstrained arthroplasty. Mod Rheumatol, 2008; 18: 465-71.

18. van der Heide HJ, de Vos MJ, Brinkman JM, et al. Survivorship of the Kudo total elbow prosthesis--comparative study of cemented and uncemented ulnar components: 89 cases followed for an average of 6 years. Acta Orthop, 2007; 78: 258-62.

19. Brinkman JM, de Vos MJ, Eygendaal D. Failure mechanis$\mathrm{ms}$ in uncemented Kudo type 5 elbow prosthesis in patients with rheumatoid arthritis: 7 of 49 ulnar components revised because of loosening after 2-10 years. Acta Orthop, 2007, 78: 263-70.

20. Spinner RJ, Morgenlander JC, Nunley JA. Ulnar nerve function following total elbow arthroplasty: a prospective study comparing preoperative and postoperative clinical and electrophysiologic evaluation in patients with rheumatoid arthritis. J Hand Surg Am, 2000; 25: 360-4.

21. Yamaguchi K, Adams RA, Morrey BF. Infection after total elbow arthroplasty. J Bone Joint Surg Am, 1998; 80: 481-91.

22. Wright TW, Wong AM, Jaffe R. Functional outcome comparison of semiconstrained and unconstrained total elbow arthroplasties. J Shoulder Elbow Surg, 2000; 9: 524-31.

23. Morrey BF, Adams RA. Semiconstrained arthroplasty for the treatment of rheumatoid arthritis of the elbow. J Bone Joint Surg Am, 1992; 74: 479-90. 